\title{
COMPARACIÓN DE SENSIBILIDAD Y ESPECIFICIDAD DE DOS TÉCNICAS DE DIAGNÓSTICO DIRECTO: KATO-KATZ-SAF Y RITCHIE-FRICK (FORMOL-GASOLINA) EN EXAMEN COPROPARASITOLÓGICO PARA LA IDENTIFICACIÓN DE ESTADIOS INFECTIVOS DE GEOHELMINTOS EN POBLACIÓN INFANTIL EN EDAD PREESCOLAR Y ESCOLAR
}

\author{
JULIO CÉSAR GIRALDO FORERO'1 ANDREA MILENA GUATIBONZA CARREÑO² \\ ${ }^{1}$ M.Sc. Esp. Docente Investigador, líder Grupo de Investigación en Parasitología \\ y Microbiología Tropical (GIPAMT). \\ Facultad de Ingeniería, Administración y Ciencias Básicas. Programa de Biología. \\ Universidad INCCA de Colombia. Bogotá, Colombia \\ ${ }^{2}$ Bióloga. Universidad INCCA de Colombia. Bogotá, Colombia \\ ${ }^{1}$ Correspondencia: Julio Cesar Giraldo. jcesargiraldo@gmail.com
}

Recibido: 17 Mayo 2017 Aceptado: 14 Junio 2017

\section{RESUMEN}

Introducción: Las parasitosis intestinales causadas por geohelmintos son consideradas un problema de salud pública que afectan principalmente a países en vía de desarrollo y son proclives a presentarse en población infantil. Los métodos diagnósticos para identificar estas entidades parasitarias son el coprológico directo y las técnicas de concentración. El método establecido por la Organización Mundial de la Salud-OMS para identificar y cuantificar geohelmintiasis es el Kato-Katz; sin embargo, este no permite realizarse en trabajos de campo con periodos de tiempo prolongados, debido a que su análisis debe llevarse a cabo en las primeras 48 horas de toma de la muestra de materia fecal.

Objetivo: Implementar el uso del fijador SAF (Acetato de sodio - Ácido acético - Formaldehído) en la técnica de Kato-Katz-OMS y evaluar la estabilidad de las estructuras de las entidades parasitarias, después de un periodo de doce meses de toma muestra y realizar comparación con la técnica de concentración Ritchie-Frick modificada (formol-gasolina), para determinar los valores de sensibilidad-S, especificidad-E, valor predictivo positivo-VPP y negativo-VPN y determinar la prevalencia del parasitismo intestinal y sus posibles variables asociadas en la comunidad infantil en edad preescolar y escolar del municipio de Chaguaní Cundinamarca.

Materiales y Métodos: Se analizaron 67 muestras correspondientes a toda la población infantil en edad preescolar y escolar del área urbana del municipio de Chaguaní Cundinamarca, las cuales fueron valoradas por los métodos de Kato-Katz-SAF y concentración de Ritchie-Frick modificada (formol-gasolina) y posterior análisis microscópico.

Resultados: La prevalencia de geohelmintiasis fue de 46,3\%, la técnica de Kato-Katz-SAF presentó valores de S, E, VPP y VPN del 100\% y la técnica de concentración de Ritchie-Frick modificada (formol-gasolina) S 60,8\%, E y VPP $100 \%$ respectivamente y VPN 62,1\%; la prevalencia para protozoos por este método fue de $65,7 \%$ hallándose agentes etiológicos patógenos y de carácter zoonótico. Se identificó que las variables onicofagia, consumo de agua sin proceso de cloración o hervido, presencia de vectores mecánicos, convivencia con animales domésticos como caninos, hacinamiento en la vivienda y uso poco frecuente de calzado, son variables de posible asociación estadística con la presencia de entidades parasitarias. Se determinó nivel de parasitemia por geohelmintiasis leve para esta población. 
Conclusión: El estudio reveló que el uso del reactivo de SAF puede implementarse en trabajos de campo prolongados conservando los estadios infectivos de las entidades geohelmínticas como: Ascaris lumbricoides, Trichuris trichiura y Uncinarias por un periodo de hasta doce meses.

Palabras clave: Geohelmintos, Kato-Katz-SAF, Ritchie-Frick (formol-gasolina), Protozoos 


\title{
COMPARISON OF SENSITIVITY AND SPECIFICITY OF TWO DIRECT DIAGNOSTIC TECHNIQUES: KATO-KATZ-SAF AND RITCHIE-FRICK (FORMOL-GASOLINA) EXAMINATION SHOWED FOR THE IDENTIFICATION OF INFECTIVE STAGES OF GEOHELMINTHS IN CHILDREN AGE PRESCHOOL AND SCHOOL
}

\begin{abstract}
Introduction: The intestinal parasitic diseases caused by geohelminths are considered a public health problem that primarily affect developing countries and are likely to occur in children. The diagnostic methods to identify these parasitic entities are the direct stool and concentration techniques. The method established by the World Organization of the Health-WHO to identify and quantify geohelmintiasis is the Kato-Katz; however, this does not occur in fieldwork with long periods of time, since analysis should be performed in the first 48 hours of the sampling of fecal matter.

Objective: Implement the use of SAF fixative (sodium acetate - acetic acid - formaldehyde) in the technique of Kato-Katz-WHO and to assess the stability of the structures of the entities parasitic, after a period of twelve months of taking shows and make comparison with the technique of modified Ritchie-Frick (formol-gasoline) concentration, to determine the values of sensitivity-S, specificity-E, predictive value PPV-positive and negative-VPN and determine the prevalence of intestinal parasitism and its possible variables associated in the infant community preschool and in schools of the municipality of Chaguanin Cundinamarca.

Materials and methods: 67 samples corresponding to the entire child population at preschool and school age of the urban area of the municipality of Chaguani in Cundinamarca, which were valued by the methods of Kato-Katz$\mathrm{SAF}$ and concentration of modified Ritchie-Frick (formol-gasoline) and subsequent microscopic analysis.

Results: The prevalence of geohelmintiasis was $46.3 \%$, the technique of Kato-Katz-SAF presented values of S, E, VPP, and 100\% and VPN technique of concentration of modified Ritchie-Frick (formol-gasoline) $60.8 \%$ S, E and VPP $100 \%$ respectively and VPN $62.1 \%$; the prevalence for protozoa by this method was $65.7 \%$ being etiologic agents pathogenic and zoonotic character. It was identified as variables onicofagia, consumption of water without boiling or chlorination process, the presence of mechanical vectors, coexistence with domestic animals such as dogs, overcrowded housing and less frequent use of footwear, are variables of possible statistical association with the presence of parasitic entities. Level of parasitemia was determined by mild geohelmintiasis for this population.

Conclusion: Study revealed that the use of SAF reagent can be deployed in field work extended to conserving the infective stages of entities as geohelminths: Ascaris lumbricoides, Trichuris trichiura and hookworms for a period of up to twelve months.
\end{abstract}

Key words: Kato-Katz-SAF; Geohelminths; Protozoa; Ritchie-Frick (formol-gasoline). 


\title{
COMPARAÇÃO DE SENSIBILIDADE E ESPECIFICIDADE DE DUAS TÉCNICAS DIAGNÓSTICAS DIRECTAS: EXAMES DE KATO-KATZ-SAF E RITCHIE-FRICK (FORMOL-GASOLINA) MOSTRADOS PARA A IDENTIFICAÇÃO DE ETAPAS INFECTIVAS DE GEOHELMINTHS EM POPULAÇÃO INFANTIL EM EDIÇÃO PRÉ-ESCOLA E ESCOLAR
}

\begin{abstract}
RESUMO
Introdução: as doenças parasitárias intestinais causadas por geohelmintos são consideradas um problema de saúde pública que afeta principalmente os países em desenvolvimento e é provável que ocorram em crianças. Os métodos de diagnóstico para identificar essas entidades parasitas são as fezes diretas e as técnica de concentração. O método estabelecido pela Organização Mundial da Saúde-OMS para identificar e quantificar geohelmintiasis é o Kato-Katz; No entanto, isso não ocorre no trabalho de campo com longos períodos de tempo, uma vez que a análise deve ser realizada nas primeiras 48 horas da amostragem de matéria fecal.
\end{abstract}

Objetivo: Implementar o uso de fixador SAF (acetato de sódio - ácido acético - formaldeído) na técnica de Kato-KatzOMS e avaliar a estabilidade das estruturas das entidades parasitárias, após um período de doze meses de exibição e exibição comparação com a técnica de concentração modificada de Ritchie-Frick (formol-gasolina), para determinar os valores de sensibilidade-S, especificidade-E, valor preditivo PPV-positivo e VPN negativo e determinar a prevalência de parasitismo intestinal e suas possíveis variáveis associadas na comunidade pré-escolar da comunidade infantil e nas escolas do município de Chaguanín Cundinamarca.

Materiais e métodos: 67 amostras correspondentes a toda a população infantil na idade pré-escolar e escolar da área urbana do município de Chaguani em Cundinamarca, que foram avaliadas pelos métodos de Kato-Katz-SAF e concentração de Ritchie-Frick modificado (formol -gasolina) e posterior análise microscópica.

Resultados: A prevalência de geohelmintiasis foi de 46,3\%, a técnica de Kato-Katz-SAF apresentou valores de S, E, VPP e 100\% e VPN de concentração de Ritchie-Frick modificada (formol-gasolina) 60,8\% S, E e VPP 100\% respectivamente e VPN 62,1\%; A prevalência de protozoários por este método foi de 65,7\% sendo agentes etiológicos patogênicos e de caráter zoonótico. Identificou-se como variáveis onicofagia, consumo de água sem processo de ebulição ou cloração, presença de vetores mecânicos, coexistência com animais domésticos, como cães, carcaça superlotada e uso menos freqüente de calçados, variáveis de possivel associação estatística com a presença de parasitas entidades. O nível de parasitemia foi determinado por geohelmintiasis leve para esta população.

Conclusão: o estudo revelou que o uso do reagente SAF pode ser implantado em trabalhos de campo estendidos para conservar os estádios infecciosos das entidades como geohelminas: Ascaris lumbricoides, Trichuris trichiura $e$ ancilostomídeos por um período de até 12 meses.

Palavras-chave: Kato-Katz-SAF; Geohelminas; Protozoários; Ritchie-Frick (formol-gasolina). 


\section{Introducción}

Las parasitosis intestinales transmitidas por geohelmintos son consideradas un problema de salud pública en el mundo, que afecta a diferentes poblaciones sin distinción de raza, edad o estrato socioeconómico; sin embargo, se presenta en mayor proporción en países en vía de desarrollo, debido a sus características geográficas, ecológicas específicas del lugar, condiciones de saneamiento básico disponible, grado de hacinamiento escolar y familiar, factores socioeconómicos y culturales $(1,2)$. Se estima que en el mundo dos mil millones de personas están infectadas con geohelmintos, de las cuales por lo menos trecientos millones sufren morbilidad severa asociada con anemia, problemas de aprendizaje, desnutrición crónica y trastornos del desarrollo y el crecimiento (3).

Estas parasitosis se producen cuando los hábitos higiénico sanitarios son deficientes e interrelacionan con los ciclos de vida de los helmintos, siendo más frecuente durante los primeros años de vida, cuando aún no se han adquirido prácticas higiénicas adecuadas para prevenirlas y la inmunidad está en proceso de desarrollo frente a diferentes tipos de agentes infecciosos (4). Sin embargo, en los niños que se encuentran en edad escolar, dependiendo el entorno en que estos se hallen, será un factor determinante para la adquisición de estadios infectivos como huevos o larvas de geohelmintos patógenos, quienes son resistentes a condiciones adversas y que vía dermal u orofecal pueden ocasionar infección (5).

En Colombia los parásitos patógenos intestinales helmintos más frecuentes son: Ascaris lumbricoides, Trichuris trichiura, Enterobius vermicularis, Necator americanus, Ancylostoma duodenale, Strongyloides stercoralis y Taenia solium (6). Estos parásitos pueden ocasionar diferentes manifestaciones clínicas dependiendo del mecanismo de daño y del tropismo específico que presenten por el sistema digestivo; entre estas se encuentran principalmente, diarrea, dolor abdominal, desnutrición, mala absorción y anemia (7).

La encuesta nacional de parasitismo intestinal en población escolar de Colombia de 2012-2014 determinó una prevalencia de geohelmintiasis de $29,6 \%$ dentro de las cuales $T$. trichiura, fue el helminto más prevalente con una frecuencia de 18,4\%, seguido de A. lumbricoides, con $11,3 \%$ y Uncinarias con $6,4 \%$, la intensidad parasitaria para estas tres entidades fue leve (8).
El diagnóstico de las infecciones parasitarias intestinales se basa ampliamente en el análisis microscópico de las muestras fecales, que incluyen montajes húmedos directos, concentrados y frotis con tinción permanente. La cantidad de formas parasitarias en muestra de materia fecal, a menudo, es escasa y difícil de detectar en preparados directos en fresco o en frotis teñidos; por lo tanto, siempre deben realizarse procedimientos de concentración (9).

La técnica de laboratorio más utilizada para hacer el diagnóstico del parasitismo ha sido el coprológico directo. Algunos autores afirman que con él se corre el riesgo de pasar por alto los casos positivos en pacientes con cargas parasitarias bajas, por lo que sugieren complementarlo con técnicas de concentración que aumentan la sensibilidad en un $30 \%$ de ellas, la sedimentación de Ritchie modificada es la más empleada en los laboratorios clínicos, porque es eficiente para detectar quistes de protozoos, huevos y larvas de helmintos, además de ser económica y sencilla en cuanto a los reactivos y equipos para su ejecución; estas características la hacen más eficiente que otras técnicas como las de Willis y Sheather que están dirigidas a grupos más reducidos de parásitos $(10,11)$.

El método diagnóstico utilizado mundialmente en encuestas parasitológicas y estudios epidemiológicos es el denominado Kato-Katz, introducido por Kato y Miura en 1954 y modificado por Katz en 1972, siendo el método recomendado por la Organización Mundial de la Salud-OMS y la Organización Panamericana de la Salud-OPS, para el diagnóstico cualitativo y cuantitativo de las geohelmintiasis humanas en este tipo de estudios (12), sin embargo esta técnica tiene como desventaja que se debe realizar el análisis microscópico, en un periodo de tiempo no máximo a 48 horas, debido a que los huevos contenidos en la muestra tienden a degradarse después de este lapso de tiempo.

Estudios realizados en Centroamérica han implementado el uso de kit comerciales para la recuperación de estadios infectivos de helmintos, como lo son el Macro-CON y el Spin-CON, las cuales son técnicas coproparasitológicas que permiten la recolección de quistes, ooquistes, huevos y larvas del sedimento de una muestra preservada de heces, mostrando una capacidad de identificación de helmintos del 99 y $100 \%$ respectivamente, en comparación con el examen tradicional de diagnóstico directo y la técnica de concentración formol éter, las cuales presentaron 68 y $97 \%$ respectivamente (13). Sin embargo estas técnicas no permiten determinar el nivel de parasitemia en la 
población debido a que impide el recuento de huevos por desconocimiento del gramaje de materia fecal utilizada.

Otro estudio realizado en Ecuador en 2016 compara la técnica de Kato-Katz con el método de flotación Mini-FLOTAC ${ }^{\circledR}$ concluyendo que esta técnica presenta una sensibilidad del $100 \%$ frente a la técnica de Kato-Katz con el $88 \%$, esto se debió a que su tiempo de ejecución es relativamente rápido en relación con la técnica de Kato-Katz (14), a pesar de los resultados, este estudio se realizó con las condiciones estandarizadas por la OMS donde la técnica de Kato-Katz no puede superar un máximo de dos horas después de preparado el montaje para su análisis microscópico, por lo cual este método no es recomendable para trabajos de campo donde las muestras no se observen en el tiempo estimado.

El objetivo de la presente investigación fue evaluar la técnica de Kato-Katz con el reactivo de SAF como fijador y lectura a los cero y doce meses de recolección de la muestra coprológica y compararla con el método de concentración Ritchie-Frick modificado (formol-gasolina) y determinar sensibilidad-S, especificidad-E, valor predictivo positivo-VPP y valor predictivo negativo-VPN de ambas técnicas en la identificación de estadios infectivos de geohelmintos y de igual manera establecer los valores de prevalencia del parasitismo intestinal y su posible asociación con variables ecoepidemiológicas en la población infantil en edad preescolar y escolar del área urbana del municipio de Chaguani-Cundinamarca.

\section{Metodología}

Se realizó un estudio descriptivo de corte transversal con toma única de muestra en la población infantil del área urbana del Municipio de Chaguaní Cundinamarca. Se comparó la sensibilidad, especificidad, valor predictivo positivo y negativo de dos técnicas coprológicas de diagnóstico directo para identificar estadios infectivos de helmintos las cuales fueron: el examen de concentración Ritchie-Frick modificado formol-gasolina y la técnica de Kato-Katz recomendada por la OMS con muestras fijadas con el reactivo de SAF y almacenadas por un periodo de doce meses.

\section{Área y población de estudio}

El estudio se realizó en el municipio de Chaguaní, el cual se encuentra localizado en el occidente del departamento de Cundinamarca y cuenta con una extensión de $142 \mathrm{Km}^{2}$. El muestreo se llevó a cabo en el área urbana, con una extensión de $0,139 \mathrm{Km}^{2}$. Este municipio hace parte de la provincia del Magdalena Centro, su cabecera se localiza a los $4^{\circ} 57^{\prime}$ de longitud norte y $74^{\circ} 36^{\prime}$ de lon- gitud oeste del meridiano de Greenwinch; con una altura de 1.050 m.s.n.m., con temperatura promedio de $24^{\circ} \mathrm{C}$. Chaguaní limita al norte con el municipio de Guaduas, al oriente con el municipio de Vianí, al sur y al occidente con el municipio de San Juan de Rioseco y el río Magdalena que lo separa del departamento del Tolima (15). Según el plan de desarrollo municipal (PDM) de 2012 a 2015 el área urbana cuenta con una población de 731 habitantes, entre ellos 150 son menores, en un grupo etario de 0 a 17 años y de estos 70 en edad preescolar y escolar (16). De igual manera cuenta con un centro de salud nivel uno, razón por la cual la mayoría de sus historias clínicas se registran en el hospital del municipio vecino que es Guaduas, desconociéndose las entidades parasitarias y las prevalencias de estas, en la población infantil en edad preescolar y escolar, propia del municipio Chaguaní.

\section{Recolección de la información}

La investigación se socializó con la comunidad participante a través de charlas educativas con el fin de dar a conocer los diferentes problemas que pueden causar los parásitos intestinales en la población infantil, en especial durante la edad preescolar y escolar.

La fase de campo se llevó a cabo en los meses de enero a marzo del año 2015, la primera etapa fue de divulgación de la información a partir de charlas educativas y material didáctico enfatizando sobre las vías de trasmisión y las consecuencias que pueden causar los parásitos intestinales en los niños. Se socializó con los adultos responsables del menor un consentimiento informado, de igual manera se contó con el asentimiento de los niños, quienes accedieron a participar voluntariamente en el estudio. Se acordó con la comunidad realizar una visita domiciliaria por parte del equipo de trabajo para la recolección de información, en la cual se empleó una ficha técnica epidemiológica estructurada, donde se incluyeron: datos personales del menor, antecedentes patológicos, costumbres alimentarias y culturales, disposición de servicios públicos en el hogar y presencia de animales domésticos como mascotas. Una vez realizada la encuesta y firmado el consentimiento por parte del acudiente responsable del menor, se suministró la información sobre la recolección apropiada de la muestra de materia fecal, posteriormente se entregó un estuche coprológico debidamente rotulado.

Se visitaron 96 domicilios de los cuales 67 realizaron entrega de la muestra de materia fecal, posterior a la recolección cada una se dividió en dos partes iguales, a la primera se le adicionó reactivo de MIF (Mertiolate, Yodo 
y Formol) y a la segunda reactivo de SAF (Acetato de sodio - Ácido acético - Formaldehído) en proporción 1:1, éstas se homogenizaron, rotularon y almacenaron a $4^{\circ} \mathrm{C}$ para su traslado al laboratorio del Grupo de Investigación en Parasitología y Microbiología Tropical - GIPAMT de la Universidad Incca de Colombia sede Bogotá D.C.

\section{Análisis por examen coproparasitológico}

Método de Kato - Katz - SAF

De la fracción preservada en reactivo de $\mathrm{SAF}$, se tomó aproximadamente uno a dos gramos de materia fecal con una espátula de madera y se colocó sobre un trozo de papel Kraft. La porción se cubrió con una malla de Nailon de $200 \mu$ de poro, con una espátula plástica se ejerció presión hasta filtrar la materia fecal, la cual se depositó sobre el orificio de la Placa Kit-OMS, con capacidad para $41,7 \mathrm{mg}$, y ésta a su vez colocada sobre una lámina porta objetos, seguidamente se retiró la misma y se cubrió la muestra con una lámina de papel celofán hidrofílico, previamente sumergida durante 24 horas en solución de glicerina con verde de malaquita al $3 \%$, la cual se presionó hasta expandir la totalidad de la misma sobre el portaobjeto; seguido de un proceso de aclaramiento por un periodo de $45 \mathrm{~min}$ se realizó la lectura ya descrita. El número total de huevos observados en el montaje se multiplicó por 24 como valor constante, para establecer la cantidad de los mismos por gramo de heces (h.p.g) y así estipular el nivel de parasitemia en la muestra.

Método de concentración Ritchie-Frick

(formol - gasolina)

Se empleó la totalidad de la fracción preservada con reactivo de MIF y se filtró a través de una gasa triple sobre un vaso de precipitado, el tamizado se colectó y se depositó en tubo cónico del $13 \mathrm{ml}$ y se centrifugó por $10 \mathrm{~min}$ a 3000 r.p.m, luego de retirado el sobrenadante, al sedimento se le agregó $10 \mathrm{ml}$ de solución de formaldehído al $10 \%$, se mezcló y se dejó reposar durante $10 \mathrm{~min}$. Transcurrido el tiempo se añadieron $3 \mathrm{ml}$ de gasolina y se agitaron los tubos mecánicamente durante 30 seg y se dejó nuevamente en reposo durante $10 \mathrm{~min}$; seguidamente la muestra se centrifugó por $10 \mathrm{~min}$ a 3000r.p.m. En el tubo cónico se observó la presencia de cuatro capas, de las cuales se eliminaron las tres superiores, el sedimento se alicuotó en tubos eppendorf con la adición de reactivo de MIF y se almacenaron a $4^{\circ} \mathrm{C}$ para su posterior lectura.
Valoración de las muestras

La lectura para la técnica de Kato-Katz-SAF (Acetato de sodio-Ácido acético-Formaldehido), se realizó con observación por duplicado y la de concentración de Ritchie-Frick (Formol- gasolina) por triplicado, en ambos casos se utilizó el objetico de 40X y el ocular calibrado con micrómetro para la medición e identificación de estadios de huevos o larvas de helmintos, como también de trofozoitos o quistes de protozoos. El examen coproparasitológico incluyo lecturas doble ciego.

\section{Tratamiento estadístico}

Para el tratamiento estadístico se emplearon tablas de contingencia de 2x2 para calcular la sensibilidad (S), especificidad (E), valor predictivo positivo (VPP) y negativo (VPN) para cada una de las técnicas de diagnóstico directo evaluadas con el empleo de las siguientes ecuaciones: $\mathrm{S}=\mathrm{a} / \mathrm{a}+\mathrm{c}, \mathrm{E}=\mathrm{b} / \mathrm{b}+\mathrm{d}, \mathrm{VPP}=\mathrm{a} / \mathrm{a}+\mathrm{b}, \mathrm{VPN}=\mathrm{a} / \mathrm{c}+\mathrm{d}$.

En el cálculo del valor de $p$, se utilizó el programa de IBM SPSS Statistics VER21PROGRAM, y así determinar la existencia de diferencias significativas entre las dos técnicas evaluadas e identificar las variables socio-ecoepidemiológicas con posible asociación estadística, con las diferentes entidades parasitarias identificadas.

\section{Resultados} Determinación de la prevalencia del parasitismo
intestinal por diagnóstico directo

Lectura de las muestras con el empleo de la técnica de Kato-Katz-SAF evaluada a los doce meses

La primera lectura de las muestras se realizó en un tiempo denominado cero donde identificaron los estadios infectivos de diferentes geohelmintos como A. lumbricoides, Uncinarias y $T$. trichiura, se almacenaron a $4^{\circ} \mathrm{C}$. Nuevamente se efectuó una segunda lectura de las muestras transcurridos doce meses de haber sido preservadas en el reactivo de SAF, con el objeto de valorar la estabilidad de las estructuras parasitarias en el fijador. Con esta técnica se halló que, para ambas lecturas, el 46,3\% (31/67) de las mismas se identificaron estadios infectivos de al menos un helminto; estableciéndose los siguientes valores porcentuales: A. lumbricoides, 22,4\% (15/67), Uncinarias $18 \%(12 / 67)$ y $T$. trichiura, $10,5 \%$ (7/67). De igual 
manera se observaron otras formas parasitarias, entre ellas S. stercolaris y E. vermicularis, con $9 \%(6 / 67)$ y $1,5 \%(1 / 67)$ respectivamente, sin embargo, para estos dos agentes, los valores no son confrontables, debido a que está técnica no es específica para calcular los niveles de parasitemia particular de ellos.

Lectura de las muestras con el empleo de la técnica de Ritchie-Frick modificado (formol-gasolina) evaluada a los doce meses

Las muestras valoradas por la técnica de concentración de Ritchie Frick (formol-gasolina) arrojó un 16,4\% $(11 / 67)$ de positivos para la presencia de al menos un helminto en particular, distribuyéndose de la siguiente manera: A. lumbricoides, 10,5\% (7/67), Uncinarias $3 \%(2 / 67)$ y $T$. trichiura, $3 \%(2 / 67)$, de igual manera se identificó la presencia de $S$. stercolaris y E. vermicularis, en un 1,5\% (1/67) para ambos casos.

Identificación de estadios infectivos de protozoos con la técnica de Ritchie-Frick modificada (formol-gasolina) evaluadas a los doce meses

Con la técnica de concentración de Ritchie-Frick modificada (formol-gasolina) se encontró al menos una entidad parasitaria para un valor porcentual de prevalencia del 65,7\% (44/67) destacándose: complejo Entamoeba histolytica/E. dispar/E. moshkouskii, 46,3\% (31/67), Entamoeba coli, 16,5\% (11/67), Giardia duodenalis, $4,5 \%$ (3/67), Blastocystis spp., $27 \%$ (18/67), Endolimax nana, 3\% (2/67) y Cyclospora spp., $24 \%(16 / 67)$.

Distribución por género del parasitismo intestinal en la población infantil

De las 31 muestras diagnosticadas positivas por la téc- nica de Kato-Katz-SAF para helmintos, evaluada a los doce meses se determinó que el 61,3\% (19/31) son del género femenino, el 32,3\% (10/31) masculino y el $6,5 \%(2 / 31)$ un grupo que se denominó indeterminado, por no contar con información como el nombre, género y edad.

Para el caso de las muestras valoradas positivas para helmintos, con la técnica de concentración Ritchie Frick modificada (formol-gasolina), se halló que el 54,5\% (6/11) corresponde al género femenino, el $36,4 \%$ $(4 / 11)$ al masculino y el $9,1 \%(1 / 11)$ al grupo indeterminado.

Para la presencia de protozoos se calculó que el $57 \%$ (25/44) pertenece al género femenino, el 38,6\% (17/44) al masculino y $4,5 \%(2 / 44)$ al grupo indeterminado.

Prevalencia de parasitismo intestinal por grupo etario en la población infantil

El rango de edad de los participantes osciló entre uno y doce años, este se distribuyó en cuatro grupos etarios con los siguientes valores porcentuales de prevalencia parasitaria: uno a cinco años $48,1 \%$, seis a diez $32,4 \%$, mayores a once $6,4 \%$, y el denominado indeterminado $12,9 \%$.

\section{Sensibilidad, especificidad, valor predictivo posi- tivo y valor predictivo negativo}

Para calcular los valores de sensibilidad-S, especificidad-E, valor predictivo positivo-VPP y valor predictivo negativo-VPN para la técnica de Kato-Katz-SAF, como las geohelmintiasis causadas por: A. lumbricoides, Uncinarias y $T$. trichiura, identificadas en lectura a los cero y doce meses se emplearon los datos relacionados en la Tabla 1. 
Tabla 1. Determinación de los valores de sensibilidad-S, Especificidad-E, valor predictivo positivo-VPP, valor predictivo negativo-VPN, para la técnica de Kato-Katz-SAF y las entidades parasitarias identificadas a los cero y doce meses

\begin{tabular}{|c|c|c|c|c|}
\hline & & \multicolumn{2}{|c|}{ Kato - Katz - SAF } & IC95\% \\
\hline Positivo & $a=31$ & $b=0$ & $S=100$ & $\mathrm{VPP}=100$ \\
\hline Negativo & $\mathrm{c}=0$ & $d=36$ & $E=100$ & $\mathrm{NPN}=100$ \\
\hline
\end{tabular}

\begin{tabular}{|c|c|c|c|c|}
\hline \multicolumn{3}{|c|}{ Ascaris lumbricoides } & $\%$ & \multirow[t]{2}{*}{ IC95\% } \\
\hline Positivos & $a=12$ & $b=0$ & $S=100$ & \\
\hline Negativo & $c=0$ & $d=55$ & $E=100$ & $\mathrm{VPN}=100$ \\
\hline
\end{tabular}

\begin{tabular}{|c|c|c|c|c|}
\hline Uncinarias & & & $\%$ & IC95\% \\
\hline Positivos & $a=15$ & $b=0$ & $S=100$ & $\mathrm{VPP}=100$ \\
\hline Negativo & $c=0$ & $d=52$ & $E=100$ & $\mathrm{VPN}=100$ \\
\hline
\end{tabular}

\begin{tabular}{|lllll|}
\hline Trichuris trichiura & \multicolumn{3}{c|}{ \% } & IC95\% \\
\hline Positivo & $\mathrm{a}=7$ & $\mathrm{~b}=0$ & $\mathrm{~S}=100$ & $\mathrm{VPP}=100$ \\
\hline Negativo & $\mathrm{b}=0$ & $\mathrm{~d}=60$ & $\mathrm{E}=100$ & $\mathrm{VPN}=100$ \\
\hline
\end{tabular}

$a=$ verdaderos positivos, $b=$ falsos positivos, $c=$ falsos negativos, $d=$ verdaderos negativos

Los valores de sensibilidad-S, especificidad-E, valor predictivo positivo-VPP y valor predictivo negativo-VPN para la técnica de concentración Ritchie-Frick modificada (formol-gasolina) y los geohelmintos identificados como A. lumbricoides, Uncinarias y Trichuris trichiura, en el proceso de lectura a los cero y doce meses se calcularon empleando los datos relacionados en las Tabla 2.

Tabla 2. Determinación de los valores de sensibilidad-S, Especificidad-E, valor predictivo positivo-VPP, valor predictivo negativo-VPN, para la técnica de Ritchie-Frick Modificada y las entidades parasitarias identificadas a los cero y doce meses

\begin{tabular}{|llllll|}
\hline \multicolumn{1}{|l}{ Ritchie-Frick Modificada } & \multicolumn{3}{c}{$\mathbf{\%}$} & IC95\% \\
\hline Positivo & $\mathrm{a}=31$ & $\mathrm{~b}=0$ & $\mathrm{~S}=60,8$ & $\mathrm{VPP}=100$ \\
\hline Negativo & $\mathrm{c}=20$ & $\mathrm{~d}=16$ & $\mathrm{E}=100$ & $\mathrm{VPN}=44,4$ \\
\hline \multicolumn{4}{|c|}{$\mathbf{\%}$} \\
\hline Ascaris lumbricoides & \multicolumn{4}{|c|}{ IC95\% } \\
\hline Positivo & $\mathrm{a}=12$ & $\mathrm{~b}=0$ & $\mathrm{~S}=54,5$ & $\mathrm{VPP}=100$ & \\
\hline Negativo & $\mathrm{c}=10$ & $\mathrm{~d}=45$ & $\mathrm{E}=100$ & $\mathrm{VPN}=81,8$ \\
\hline
\end{tabular}

\begin{tabular}{|c|c|c|c|c|}
\hline Trichuris & & & $\%$ & IC95\% \\
\hline Positivo & $a=7$ & $b=0$ & $\mathrm{~S}=58,3$ & $\mathrm{VPP}=100$ \\
\hline Negativo & $c=5$ & $d=55$ & $E=100$ & $\mathrm{VPN}=91,6$ \\
\hline
\end{tabular}


Cálculo del valor de Chi-Cuadrado, p calculado para ambos métodos y determinación del nivel de parasitemia por la técnica de Kato-Katz-SAF

Valor de p y significancia estadística para las dos técnicas

Se tabuló una base de datos con la información registrada en la encuesta estructurada, los valores hallados para cada técnica y las entidades parasitarias. El valor de $p$ se determinó con el empleó del programa de IBM SPSS Statistics ${ }^{\mathrm{VER} 21 \mathrm{PROGRAM}}$.

Al confrontar las dos técnicas se observó que se presentaron diferencias significativas con base en los valores de $p \leq 0,05$ para Kato-Katz-SAF frente a Ritchie-Frick modificada (formol-gasolina), permitiendo inferir que la primera presenta mayor sensibilidad.

Valores de p y significancia estadística para geohelmintos y Determinación de los niveles de parasitemias por la técnica de Kato-Katz-SAF
De igual manera se calculó el valor de $p$ para cada técnica, y para las entidades geohelmínticas identificadas, las cuales no presentaron diferencias estadísticamente significativas, corroborando que cualquier geohelminto de los observados es identificable indiscriminadamente por ambos métodos; sin embargo al determinar el valor de $p$ para cada uno de los agentes etiológicos hallados entre sí con las respectivas técnicas, estas si presentaron diferencias significativas; al igual que al calcular los niveles de parasitemia por gramo de muestra, mediante la operación de conteo del número de huevos hallados por montaje y multiplicarlo este, por el valor del factor constante de 24, permitió inferir el número de estos por gramo de heces (h.p.g), método de conteo que sólo es aplicable para tres entidades geohelmínticas como como son: A. lumbricoides, Uncinarias y Trichuris trichiura, limitante que presenta la técnica independientemente de que se emplee o no el fijador SAF. Los valores se presentan en la Tabla 3.

Tabla 3.Valores de p calculado para las técnicas de Kato-Katz-SAF y Ritchie- Frick- Modificada (Formol-Gasolina) y niveles de parasitemia hallados en la población en edad preescolar y escolar de la población de Chaguaní Cundinamarca

\begin{tabular}{lcl|}
\hline & Kato-Katz-SAF & Ritchie-Frick ( formol-gasolina) \\
\hline & $\boldsymbol{P}$ & $\boldsymbol{P}$ \\
Ascaris lumbricoides & 0,00 & 0,00 \\
Uncinarias & 0,00 & 0,00 \\
Trichuris trichiura & 0,00 & 0,00
\end{tabular}

\begin{tabular}{|c|c|c|c|c|c|c|}
\hline \multicolumn{3}{|c|}{ Rango de Nivel de infección (h.p.g)-OMS } & \multicolumn{4}{|c|}{ Nivel Infección Población Estudiada } \\
\hline & Leve & Moderada & Severa & $\begin{array}{l}\mathbf{N}^{\circ} \text { Muestras } \\
\text { Positivas }\end{array}$ & $\begin{array}{l}\text { Rango } \\
\text { (h.p.g) }\end{array}$ & $\begin{array}{l}\text { Nivel } \\
\text { Parasitemia }\end{array}$ \\
\hline Ascaris lumbricoides & $1-4.999$ & $5.000-49.999$ & $\geq 50.000$ & 15 & $24-168$ & Leve \\
\hline Trichuris trichiura & $1-999$ & $1.000-9.999$ & $\geq 10.000$ & 7 & $24-192$ & Leve \\
\hline Uncinarias & $1-1.999$ & $2.000-3.999$ & $\geq 4.000$ & 12 & $24-120$ & Leve \\
\hline
\end{tabular}

Relación de los valores de $p \leq 0,05$ con posible asociación y significancia estadística, para las entidades parasitarias halladas en el estudio
Los valores de $p$ calculado de las variables con posible significancia estadística con las entidades parasitarias halladas en el estudio causadas por helmintos y protozoos se presentan en la Tabla 4. 
Giraldo J, Guatibonza A.

Tabla 4. Valores de p calculado con significancia estadística de las variables con posible asociación al parasitismo intestinal en la población evaluada y la técnica empleada.

\section{Kato-Katz-SAF}

Agente etiológico

Ascaris lumbricoides

Uncinarias

Trichuris trichiura

Strongyloides stercoralis

\section{Variable asociada \\ Onicofagia}

Consumo de agua sin procesos de Hervido y cloración

Presencia de vectores mecánicos en la vivienda

Ninguna

Hacinamiento en la vivienda

Valor de $p$ IC95\% 0,002

0,004

0,003

0.003

\section{Ritchie-Frick (formol-gasolina)}

\section{Agente etiológico}

E. histolytica/E. dispar/E. moshkouskii

Entamoeba coli

Giardia duodenalis

Blastocystis spp

Endolimax nana

Cyclospora spp

\section{Variable asociada}

Presencia de mascotas en la vivienda

Ninguna

Presencia de mascotas en la vivienda

Ninguna

Consumo de agua sin procesos de Hervido y cloración

Ninguna
Valor de $p$ IC95\%

0,009

0,002

0,002

\section{Discusión}

Las técnicas coproparasitológicas para el diagnóstico de parásitos intestinales, han sido modificadas en los últimos 20 años, proporcionando mejoras en el momento de la identificación, costo, transporte y simplicidad del proceso. Los métodos de sedimentación por concentración son considerados los más opcionales al momento de la identificación de estadios infectivos tanto de helmintos como de protozoos, teniendo como ventaja que se pueden preservar por largos periodos de tiempo y son los mejores en el momento de un análisis a nivel cualitativo. Sin embargo, estos no permiten identificar un nivel de parasitemia de manera cuantitativa.

El método estandarizado para determinar niveles de parasitemia ha sido desde hace más de 30 años la técnica de Kato-Katz, ésta permite realizar un conteo y cuantificar los huevos presentes por gramo de heces (h.p.g) suministrando información sobre niveles de parasitemia ya sean: leves, moderados o severos; sin embargo, la desventaja radica en que su lectura se debe realizar en un tiempo menor a las 48 horas de toma de la muestra.

Las soluciones de fijadoras han sido consideradas útiles en el momento de preservar las muestras sobre todo cuando se hacen estudios de investigación en campo o en zonas muy alejadas o de difícil transporte para el traslado de estas. La solución de SAF contiene acetato de sodio y ácido acético que en conjunto funcionan como un sellador para preservar y estabilizador del $\mathrm{pH}$ de la muestra, de igual forma contiene formol el cual es agente fijador que coagula las proteínas permitiendo que los huevos de los helmintos sean permeables (17), sin embargo se desconoce con exactitud cuánto tiempo en preservación puedan mantenerse las muestras, sin que se pierda la utilidad de la técnica de Kato-Katz en la lectura.

El estudio realizado en la inspección La Virgen del municipio de Quipile Cundinamarca por Fernández et al. (2015), evaluó la utilidad del reactivo de SAF a los seis 
meses y reveló una prevalencia de geohelmintiasis de $14,3 \%$ dentro de la cual el porcentaje encontrado de $A$. lumbricoides, fue 7,8\%, T. trichiura, 13,6\% y Uncinarias de $0 \%$. En el presente estudio se prolongó el uso del reactivo de SAF para un periodo de doce meses, preservándose las características morfométricas de los estadios de huevos de las tres entidades geohelmínticas identificadas; infiriéndose de esta manera que el reactivo de SAF le confiere estabilidad a las muestras y por tal motivo es recomendable para suplementar la técnica de Kato-Katz en la identificación de las formas infectivas de geohelmintos en trabajos de campo prolongados.

Al realizar la comparación entre el método de KatoKatz-SAF con lectura a los doce meses y el de concentración por Ritchie-Frick modificado (formol-gasolina); el cual es empleado normalmente para el diagnóstico de entidades parasitarias, se identificaron indiscriminadamente los geohelmintos anteriormente mencionados. Según Aquino et al. (2012), las técnicas de sedimentación tienen la ventaja de recuperar un amplio número de entidades parasitarias, pero como desventaja, es que la muestra presenta un elevado número de detritos, lo cual hace que se dificulte la observación de estadios infectivos y de esta manera se generen falsos negativos (13). Los resultados obtenidos en esta investigación son concordantes con lo publicado por otros autores en cuanto a que refieren que los valores de prevalencia para los geohelmintos comparando la técnica de concentración Ritchie-Frick, es inferior a los determinados por el método de Kato-Katz-SAF.

Al comparar los valores de S, E, VPP y VPN calculados para el Kato-Katz-SAF frente a los del Ritchie-Frick modificado (formol-gasolina) estos fueron superiores, por lo que se deduce, que la técnica de Kato-Katz, no permite margen de error en el diagnóstico de falsos negativos. De igual manera, los valores para las entidades geohelmínticas identificadas por la prueba de Kato-Katz-SAF superaron a los de Ritchie-Frick (formol-gasolina), corroborando que ésta posee una confiabilidad del 100\% en el momento de identificar estadios infectivos para las tres entidades parasitarias.

Comparando el presente estudio, con el realizado en la inspección La Virgen, municipio de Quipile Cundinamarca por López et al. (2013), en donde evaluaron la S y E entre la técnica de Kato-Katz de Brasil con el método de Ritchie-Frick (formol-éter), se concluyó que el primero presenta mayor sensibilidad para las tres parasitosis: A. lumbricoides, Uncinarias y $T$. trichiura, de $79 \%, 97 \%$ y $96 \%$ respectivamente, en tanto que el método de concentración (formol- éter), presentó valores menores para los tres geohelmintos, siendo estos de $56 \%, 24 \%$ y $11 \%$ respectivamente (18).

Así mismo el estudio realizado en la escuela Santa María de la Cruz, localizada en el nororiente de la ciudad de Medellín por Restrepo et al. (2013), Evaluó tres técnicas de diagnóstico coproparasitológico siendo estas, examen directo, concentración o Ritchie-Frick y Kato-Katz el establecido por la OMS, y quienes reportaron para las tres pruebas, una $\mathrm{S}$ en la identificación de A. lumbricoides, T. trichiura y Uncinarias, valores porcentuales de $94,7 \%, 82,3 \%$ y $85,7 \%$ respectivamente, lo que les permitió concluir a los autores que tal similitud en los resultados se presentaron debido a que los niveles de parasitismo en la población estudiada, se halló en el rango de severo (h.p.g),facilitando la presencia e identificación de los estadios infectivos para los tres geohelmintos por cualquiera de los tres métodos a evaluados. Es de aclarar que esta investigación empleó la técnica tradicional de Kato-Katz sin la implementación de un fijador para las muestras (19).

Las dos técnicas confrontadas en el estudio de Chaguaní, revelaron que los valores de $p \leq 0,05$ para cada una presentaron diferencias significativas entre sí; sin embargo, en la identificación de las parasitosis de manera individual no se hallaron tales, por lo cual los dos métodos son óptimos en la identificación de cualquiera de las tres geohelmintiasis, pero es de tener en cuenta lo manifestado por Gabrie et al. (2012) en la investigación que realizó en Honduras empleando la técnica de KatoKatz-OMS, y quien si hallo diferencias significativas en la identificación de las Uncinarias, y esto lo atribuyó a que el proceso de aclaramiento y de lectura de las muestras fue superior a los 40 minutos y aunado a esto, se llevó acabo en temperaturas que oscilaron entre 25 y $28^{\circ} \mathrm{C}$, lo que repercutió posiblemente en la deshidratación de los huevos de ancililostómidos, dificultando así la observación e identificación de los mismos (20). Las condiciones de laboratorio en el cual se evaluaron las muestras del estudio de Chaguaní, entre ellas la temperatura, que no superó los $20^{\circ} \mathrm{C}$, permitiendo que las muestras se preservaran un mayor periodo de tiempo (60 a 90 min en proceso de aclaramiento); sin sufrir ninguna alteración en la morfología de los huevos en especial para las Uncinarias, pero si es de aclarar que se apreció una ligera pérdida de turgencia de la membrana de estos, pero en ningún momento afectó la identifi- 
cación de los mismos en el examen microscópico, sin embargo, no se descarta que periodos excesivos en el proceso de aclaramiento, inclusive a baja temperatura se deshidraten totalmente.

Se destaca que la técnica de concentración Ritchie-Frick es utilizada para calcular la prevalencia tanto de helmintos como de protozoos en estudios ecoepidemiológicos, siendo el método de elección, con excelente eficiencia para la identificación de estadios infectivos de agentes patógenos como G. duodenalis, complejo E. histolytica/ E. dispar/E. moshkouskii, E. coli, y B. hominis, entre otros (11) como lo enfatiza el estudio realizado en 2013 en una comunidad de la ciudad de Medellín, quien reporto una prevalencia del $69,6 \%$ para protozoos en general, manifestando que la efectividad en diagnóstico radica que no se requiere la observación inmediata de la muestra, ni el empleo de materiales y equipos sofisticados (10); esta aseveración es concordante con los resultados observado en la presente investigación, donde se halló una prevalencia de $65,7 \%$, identificándose los agentes etiológicos mencionados anteriormente y de los cuales los se destacó por ser el más prevalente el complejo E. histolytica/E. dispar/E. moshkouskii, y el menor proporción E. nana. Navone et al. (2005), hacen referencia en su publicación que en ocasiones el diagnóstico de algunas estructuras de protozoos suele ser difícil, debido a que en el proceso de centrifugación los quistes se deforman y se presentan como residuos en el momento de la observación como puede ser el caso de elevado pleomorfismo observado para Blastocystis spp.,(21); lo que probablemente se puede atribuir a las muestras evaluadas para la población de Chaguaní, donde en la mayoría de los casos solo fue posible la identificación de este parásito mediante procesos de medición con micrómetro calibrado y confrontación con la literatura.

Al evaluar las entidades parasitarias de manera individual y las posibles asociaciones que puedan presentar con las variables incluidas en la ficha epidemiológica, se observó que para el caso de A. lumbricoides, se obtuvo significancia estadística para $p \leq 0,05$ con la variable de onicofagia. Estudio realizado en ciudad de Valles México por Rodríguez et al. (2004), no encontró relación con factores de posible riesgo con la presencia de este helminto, aunque si evidenció que fue prevalente en los infantes de uno a cinco años de edad (22). La publicación de Pinto y Peredo (2010), del estudio realizado en Ivirgarzama, Bolivia, demostró que niños que presentaron algún grado de desnutrición, cuadros de anemia y portadores de otras parasitosis, estaban en mayor grado de predisposición para presentar infección por este geohelminto (23) . Sin embargo, es bien sabido que la vía de transmisión de esta entidad parasitaria es fecal-oral y según lo reportado por Chacón et al. (2007), el cuidado higiénico de los niños en sus primeros años de vida, depende de personas mayores y si esta no se realiza de forma adecuada, se incrementa la probabilidad de infección por factores como: el no lavado de manos después de hacer uso del sistema sanitario, contacto directo con suelos, geofagia y fecalismo al aire libre entre otros (24),esto se puede relacionar de igual manera con la variable de onicofagia que presentan los niños evaluados en el municipio de Chaguaní y sumado a esto el no lavado de manos de los mismos, logrando acrecentar la probabilidad en la transmisión de esta entidad parasitaria.

Para el caso de Uncinarias, según lo reportado por Carrada (2007) los factores más frecuentes para que se presente este helminto, son el hábito de caminar descalzo sobre la tierra, las actividades de juego al aire libre y el contacto directo con esta, el no lavado de los alimentos como frutas y verduras antes de consumirlos y el fecalismo a ras de piso, sobre todo en poblaciones que presentan un estado socioeconómico bajo (25); En tanto que el estudio de Peredo et al. (2012), realizado en Puerto Villarroel-Cochabamba Bolivia menciona que el consumo de agua no potable es un factor incidente para la presencia de Uncinarias (26), con respecto a la actual investigación, se obtuvo diferencias estadísticamente significativas para las variables como consumo de agua sin proceso de hervido o cloración y presencia de vectores en la vivienda con respecto a la infección por ancilostomidos; otro aspecto relevante a tener en cuenta son las condiciones de la vivienda como factor de riego determinante en la adquisición de infecciones parasitarias, como es el hecho de la presencia de vectores mecánicos entre ellos moscas, cucarachas y roedores según lo plantea la publicación de Espinosa et al. (2011), en el estudio realizado en la ciudad de Maracaibo Venezuela (27); situación similar se observó con esta variable denominada presencia de vectores mecánicos en la vivienda, que permite sugerir que dicha condición puede estar relacionada con la transmisión de helmintos en general para la población infantil del área urbana del municipio de Chaguaní.

En el caso de S. stercoralis, se apreció significancia estadística para la variable hacinamiento de vivienda con un valor de $p \leq 0,05$, según lo reportado por Igual y Domínguez (2007), en investigación realizada en la ciudad de Valencia España, un factor epidemiológico asociado a la presencia de esta entidad es realizar actividades con 
los pies descalzos en tierras fangosas durante largos periodos de tiempo, debido a que este parásito desarrolla parte de su ciclo de vida en estas, por su condición de geohelminto y cuya vía de infección es dermal (28); sin embargo, Carrada (2008), menciona que en medios con condiciones básicas insatisfechas, hacinamiento en el hogar, fecalismo al aire libre e insalubridad, favorecen sin duda la aparición de esta parasitosis (29), esta premisa se corrobora en la población evaluada donde la condición de hacinamiento en la vivienda presentó significancia estadística frente a esta entidad parasitaria.

A pesar de que las técnicas de Kato-Katz-SAF y Ritchie-Frick modificada (formol-gasolina) no son las específicas para la identificación de E. vermicularis, se encontró una prevalencia del 3,2\%. Sin embargo, un estudio realizado en la población de Chaguaní simultáneo a éste en 2015, el cual implementó la técnica de referencia de Graham para la identificación de éste agente etiológico, halló una prevalencia de $27,2 \%$ y las variables que presentaron significancia estadística con valor de $p \leq 0,05$ fueron: presencia de vectores en la vivienda, fómites y sistema beneficiado "Sisben", de igual manera resaltan que las variables relacionadas con el parasitismo por esta entidad son: viviendas en condiciones inadecuadas, deficiencias en el manejo de excretas, no poseer agua potable y ausencia de tratamiento antiparasitarios (30).

Para los parásitos del grupo de los protozoos se identificaron algunas variables con significancia estadística con valor de $p \leq 0,05$, siendo éstas el complejo $E$. histolytica/E. dispar/E. moshkouskii, y G. duodenalis, hallándose asociación con la presencia de animales domésticos en la vivienda, entre ellos caninos y felinos. Para la primera entidad autores como Xíménez et al. (2007) y López et al. (2008), determinaron que los factores de riesgo con mayor asociación son el consumo de agua sin ningún tipo de tratamiento, la contaminación del suelo con heces, saneamiento básico ambiental deficiente por mal manejo de los productos de desecho humano o de animales domésticos, el clima, factores socioeconómicos y culturales, y sumado a esto la susceptibilidad del huésped $(31,32)$. Sin embargo, según lo publicado por Ospina et al. (2012); y Giraldo et al(2017); una variable a tener en cuenta en la adquisición de la segunda parasitosis, es el estar en contacto con animales domésticos o sus excretas favoreciendo la infección y transmisión de la giardiasis por su carácter zoonótico y por ser frecuente en caninos; otro aspecto de consideración es si la crianza y manutención se da en condiciones no tecnificadas, como suele suceder para el caso de los porcinos especialmente en aquellas comunidades de escasos recursos y que habitan en zonas marginales donde las condiciones básicas son insatisfechas, siendo esto otro posible factor de riesgo en especial para la población infantil de adquirir infecciones parasitarias, debido a su participación directa o indirecta en el sustento de los mismos, $(33,34)$.

Teniendo en cuenta lo expuesto por los autores citados, los participantes de la actual investigación que manifestaron convivir con animales domésticos y entre ellos caninos, se les identificó al menos una infección causada por un protozoo, llamando la atención G. duodenalis., al igual que lo publicado por Calchi et al. (2014), en un estudio realizado en la ciudad de Maracaibo Venezuela donde se discutió que la giardiasis se disemino principalmente de persona a persona, pero que algunos animales como caninos, felinos, roedores domésticos y silvestres, pudieron ser los reservorios de G. duodenalis, a partir de los cuales se pudo originar la infección en los humanos por ser este agente etiológico causante de zoonosis (35); como también lo manifiesta el estudio realizado en caninos de la zona urbana del municipio de Coyaima, Tolima por Gonzáles y Giraldo (2012), donde se demostró la presencia de esta entidad en las mascotas evaluadas (36), y además teniendo en cuenta lo argumentado por Alvarado y Vásquez (2006), en estudio que realizaron en la población infantil de Guapi Cauca, donde argumentan que es de amplio conocimiento que este agente parasitario zoonótico, además de causar cuadros diarreicos recurrentes, malnutrición y avitaminosis infantil, es una de las patologías más frecuentes en la población preescolar, dado su grado de vulnerabilidad (37).

Un estudio realizado en caninos domiciliados de la zona urbana del municipio de Chaguaní en 2015 determinó que el parasitismo intestinal fue prevalente para entidades helmínticas en un 30,5\%, entre las cuales las Uncinarias y Strongyloides spp., fueron del $17 \%$ y $12 \%$ respectivamente y para protozoos del $29 \%$ siendo Entamoeba spp., y Giardia spp., 23\% y 10\%, aunque los propietarios de las mascotas manifestaron el tener un buen cuidado en la higiene, cumplir oportunamente con los esquemas de vacunación y suministrar los tratamientos de desparasitación correspondientes (38); sin embargo, no es descartable que se pueda presentar trasmisión de la infección de estos parásitos a la población infantil del estudio; evento similar al realizado en la ciudad de Bogotá D.C. en la localidad de Teusaquillo en 2015, que a pesar de las buenas condiciones médico-veterinarias de los animales valorados, se observó la presencia de parásitos intestinales en 
los caninos evaluados, quienes presentaron una prevalencia del 9\%, corroborando que de la condición socioeconómica que poseen los propietarios permiten que estos tengan mayor accesibilidad a los esquemas de tratamientos antiparasitarios, razón por lo cual las mascotas tienden a ser menos propensas para el contagio con alguna entidad parasitaria y de esta manera disminuye el riesgo de que se presenten infecciones de tipo zoonótico (39); pero de igual manera Díaz et al 2015 en el estudio realizado en parques de la ciudad de Tunja manifiesta que tanto la población infantil como los caninos de compañía de buena condición de manutención, pueden sufrir reinfecciones cuando sus propietarios los llevan a lugares de recreo como zonas verdes y parques públicos ya que en estos se pueden hallar estadios infectivos excretados por animales cuya condición de no domiciliados los hace proclives a estar parasitados y por su condición no reciben ningún tipo de tratamiento (40).

Varios autores manifiestan que G. duodenalis, es frecuente en la población infantil causando problemas de mal nutrición y cuadros diarreicos y se presenta incluso en comunidades que poseen buenas condiciones de saneamiento ambiental y las básicas satisfechas, como lo reporta el estudio de Castañeda et al. (2015), realizado en un jardín infantil público de la localidad de Chapinero en Bogotá D.C. donde identificó una prevalencia del $7,4 \%$, argumentando que el posible carácter zoonótico de este agente etiológico en especial con mascotas como caninos, sea el causante de la infección en los niños de edad preescolar por el contacto directo que puedan tener con estas en su lugar de residencia (41).

Según la Encuesta Nacional de Parasitismo Intestinal en población escolar, Colombia, 2012-2014, la prevalencia de G. duodenalis, a nivel nacional se presenta en un $15,4 \%$ y para la región andina en $11,2 \%$, lo que connota la frecuencia de este agente etiológico en comunidades de áreas rurales y urbanas en edad preescolar y escolar sin distingo de género (8).

E. nana, presentó significancia estadística para las variables consumo de agua sin proceso de hervido o cloración y el uso de calzado poco frecuente; un estudio realizado en una comunidad rural de Venezuela por Devera et al. (2003), sostiene que el hecho de presentar contaminación en acueductos o que las fuentes de aguas para consumo humano, no sean tratadas adecuadamente, al igual que el consumo directo de la llave sin ningún proceso de floculación y cloración, es un factor relevante que favorece la presencia de agentes parasitarios, siendo con mayor frecuencia protozoos (42), evento que es similar al de la población estudiada donde se observó que aquellos que presentaron al menos una entidad parasitaria, el 29,5\% manifestaron que a pesar que es de su conocimiento que no poseen fuente de agua tratada, realizan consumo directo de esta sin procesos de hervido, cloración o filtrado.

La variable condición de la vivienda según lo manifestado por Tarín et al. (2015), en estudio realizado en una población infantil en Florencia Caquetá, las características y tipo de piso de la vivienda puede ser un factor de riesgo, para la infección con algún agente parasitario. La carencia de pisos construidos en materiales como cerámica o baldosa, que garantizan una limpieza adecuada aminorarían este factor, caso contrario los de tierra o arcilla que puede favorecer el desarrollo de diferentes agentes parasitarios como protozoos o geohelmintos por actividad de geofagia de los menores, o infección por vía cutánea, en especial en aquellas personas que con hábito de no uso frecuente de calzado (43), evento que pude ser atribuible también en el presente estudio donde los participantes manifestaron tener como conducta recurrente el caminar descalzos y algunos de estos presentaron parasitosis al menos por una entidad ya fuese por Uncinarias, $A$. lumbricoides, S. stercoralis o T. trichiura. A pesar de no hallarse significancia estadística con la variable contacto directo con tierra, no se descarta que posiblemente ésta, sea una condición que favorezca la infección con los agentes etiológicos mencionados.

Los niveles de parasitemia por helmintos como: Uncinarias, A. lumbricoides, y T. trichiura, en la población evaluada, mediante el cálculo de cantidad de huevos por gramo de heces osciló entre 24 y 192h.p.g; de acuerdo a lo establecido por la OMS, ésta se encuentra en condición baja o leve para los tres; lo que permite asegurar que la técnica de Kato-Katz-SAF en comparación con la de concentración formol - gasolina, además de ser más sensible, a su vez permite cuantificar los niveles de parasitemia; como lo argumenta el estudio realizado por Sorto et al. (2015), en el Salvador, donde estipula que dichos niveles puede obedecer a distintos factores socioeconómicos que se presenten en la población, es decir, si el área estudiada presentaba condiciones de saneamiento básico en buen estado como: cobertura de sistema de alcantarillado en más del $90 \%$ de las viviendas, controles satisfactorios en salud y educación, los niveles porcentuales de parasitemias serían en menor proporción a los de aquellas poblaciones que no cuentan con igual condición de saneamiento básico, concluyendo que para este país, la prevalencia e intensidad de la infección por geohelmintos como A. lumbricoides, y Uncinarias fue elevada y en menor proporción para 
T. trichiura, (44). De igual manera, el estudio realizado por Londoño et al. (2005); en el municipio de Santo Tomás, Atlántico, presentó niveles elevados de parasitemia para estas tres helmintiasis y los autores lo atribuyeron a que el municipio se asemeja más a una zona rural y no cuenta con un saneamiento básico favorable (45); en sentido contrario, el estudio realizado por Hernández et al (2015); en un jardín infantil de la ciudad de Bogotá D.C; no arrojó valores de parasitemias causadas por geohelmintos, esto debido a que los participantes gozaban de buenos hábitos higiénicos y residen en viviendas con condiciones básicas satisfechas (7).

El 90\% de la población evaluada del área urbana del municipio de Chaguaní de acuerdo a la encuesta estructurada manifestaron, que las viviendas están dotadas con servicios públicos como acueducto, alcantarillado, energía y recolección de desechos entre otros; sin embargo, se presentan lugares de residencia con ausencia de alguno de estos o cuya condición no es óptima, en especial el suministro de agua lo que permite inferir por qué los niveles de parasitemias causados por helmintos son leves. La Encuesta Nacional de Parasitismo Intestinal en población escolar, Colombia, 2012-2014 identificó una prevalencia de helmintiasis para la región andina de 7,82\% distribuidos de la siguiente manera: A. lumbricoides, $3,2 \%, T$. trichiura, 4,3\%, Uncinarias $1 \%$, S. stercolaris, $0.3 \%, E$. vermicularis, $0,8 \%$ e Hymenolepis nana, $0,4 \%$ concluyendo que los departamentos de esta región presentan bajo riesgo de infección para estas y sus niveles son leves en aquellas poblaciones que se identificaron, siendo esto concordante con lo hallado en la comunidad evaluada en Chaguaní Cundinamarca (8).

Varios estudios realizados de por diferentes autores en municipios de la región andina registran valores de prevalencias para helmintos como: Fernández et al. (2007); en el corregimiento inspección La Virgen del municipio de Quipile Cundinamarca 18,18\%; Cardona et al. (2013) en barrios marginales de la ciudad de Medellin 24,6\%. Giraldo et al. (2005); en el área urbana del municipio de Armenia 1,52\%. Mayorga (2003) en Suaita Santander 17,2\%, Rodríguez (2014); en el municipio de Soracá Boyacá 2,33\%, Alvarado et al. (2015); en el municipio de Coyaima Tolima 16\%; entre otros. Los parásitos más prevalentes en estos estudios fueron $A$. lumbricoides, $T$. trichiura y Uncinarias exceptuando los estudios de Armenia Quindío y Soracá Boyacá donde no se reportaron infecciones para este último $(3,10,46-49)$; Lo que permite destacar la concordancia de estas tres helmintiasis quienes fueron las más frecuentes en la población evaluada del municipio objeto del estudio.
Se reitera que previa a esta investigación, tanto la Secretaría Municipal como la Departamental no contaban con registros para infecciones causadas por agentes parasitarios en la población infantil en edad preescolar y escolar del área urbana de Chaguaní, siendo este estudio el primer reporte que brindó información epidemiológica en cuanto a cuales son las entidades más prevalentes y las posibles variables asociadas a estas; lo que hizo necesario que se estableciera un plan que incluyera alternativas de educación, desparasitación, prevención y control, para contrarrestar el impacto que pudieran ocasionar las infecciones parasitarias como las causantes de problemas de salud pública en la comunidad y en especial en la población infantil.

\section{Conclusiones}

La implementación de la técnica de Kato-Katz con fijador SAF brinda una herramienta valiosa para trabajos en campo, proporcionando la posibilidad de conservar las muestras y poder realizar su lectura sin que se alteren los resultados hasta un periodo de doce meses, gracias a que este fijador brinda estabilidad a las formas de los estadios infectivos de los helmintos, lo que la hace recomendable para ser implementada en futuras investigaciones para trabajos en campo, si las muestras se deben almacenar por periodos prolongados; demostrando ser más estable en comparación con el método de Kato-Katz tradicional recomendado por la OMS.

La técnica de Kato-Katz-SAF presenta valores de S, E, VPP y VPN del $100 \%$ confiriéndole fiabilidad, sin que se afecte la capacidad de detección las estructuras infectivas, en aquellas muestras cuyos niveles de parasitemia sean leves. La técnica de concentración Ritchie-Frick modificada (formol -gasolina) presentó: S 61\%, VPN $62,1 \%$, E y VPP del 100\%, aunque con este método se corre el riesgo de incurrir en diagnósticos falsos negativos para el caso de helmintos en general, los métodos de concentración son los de elección recomendados por la OMS, para la identificación de estadios infectivos de protozoos, ventaja que presenta frente a los de recuento que sólo permiten identificar fase de huevos o larvas de helmintos, además la sustitución en la técnica del éter por gasolina aminora el costo, sin el detrimento de las ventajas del método tradicional de formol-éter.

Las variables ecoepidemiológicas que presentaron valores de $p$ con significancia estadística y posible asociación con infecciones parasitarias causadas por helmintos y protozoos fueron: onicofagia, consumo de agua no tratadas con procesos de hervido, filtración o cloración, 
hacinamiento en los cuartos dormitorios de la vivienda, presencia de vectores mecánicos, poseer animales de compañía como caninos y felinos en el hogar y el hábito de caminar descalzo; estas variables también han sido destacadas por otros autores argumentando que es bien sabido que los niveles de parasitemia están directamente relacionados con las condiciones de saneamiento básico que se presente en las comunidades evaluadas, si bien el $90 \%$ de la población del área urbana del municipio de Chaguaní, Cundinamarca, tiene cobertura y satisfacción de las condiciones básicas de saneamiento ambiental, es de anotar que se identificaron parásitos con potencial patógeno y carácter zoonótico.

\section{Agradecimientos}

Al Grupo de Investigación en Parasitología y Microbiología Tropical- GIMPAT, adscrito al Programa Profesional de Biología de la Universidad INCCA de Colombia y a la comunidad del Municipio de Chaguaní por su participación en el proyecto.

\section{Declaración de conflicto de Intereses}

Los investigadores manifestamos que esta investigación no presenta ningún conflicto de interés, que todos los participantes en el estudio fueron debidamente notificados, que las muestras biológicas y la información que este contiene se mantuvo con estricta reserva y tratamiento bioético.

\section{Fuente de financiación}

Este estudio fue financiado por la Universidad INCCA de Colombia aprobado en la Convocatoria interna 2013/2013-10 y desarrollado a partir del año 2015, por el Grupo de investigación de Parasitología y Microbiología tropical, GIPAMT de la Universidad INCCA de Colombia.

\section{Referencias}

1. Wordl Healt Organization. Soil-Transmitted Helminthiases: Eliminating soil-Transmitted Helminthiases as a public health problem in children [Internet]. WHO Library Cataloguing in Publi;2012. Disponilble en: http://apps.who.int/iris/bitstre am/10665/44804/1/9789241503129_eng.pdf

2. Cañavate C, Cuadros J, Martín-Rabadán P, Martinez R. El laboratorio de microbiología ante las enfermedades parasitarias importadas.EL SEVIER.2010;28(10):719-25. Doi: http://dx.doiorg/10.1016/j.eimc.2010.03.013

3. Fernández J, Reyes P, Moncada L, López M, Cháves M del P, Knudson A, et al. Tendencia y Prevalencia de las Geohelmintiasis en La Virgen, Colombia 1995-2005.
Rev Salud Pública.2007;9(2):289-96. Doi:http://dx.doi. org/10.1590/S0124-00642007000200012

4. Armengol CP, Astolfi CA, Ontiveros JMÚ, Guevara DC, Rojas M De, Lozano C. Epidemiología del parasitismo intestinal infantil en el valle de Guadalquivir, España. Departamento de Microbiología y Parasitología. Universidad de Sevilla. Rev Esp Salud Publica.1997;71(6):547-52. Disponible en: http://www.msssi.gob.es/biblioPublic/ publicaciones/recursos_propios/resp/revista_cdrom/ VOL71/71_6_547.pdf

5. Gonçalves AQ. Epidemiología y diagnóstico de Calodium hepaticum y parásitos intestinales en áreas remotas amazónicas. Universidad de Barcelona. Tesis de grado.2014;pag94.Disponible en: http://www.tdx.cat/handle/10803/134691

6. Giraldo JC, Chala DM, Vásquez LR. Sintomatología asociada a neurocisticercosis en personas del área urbana y periurbana del municipio de Coyaima del Estado del Tolima. Rev Med UMNG.2016;24(2):8-22. Doi: http://dx.doi.org/10.18359/rmed.2626 Disponible en: $\quad$ http://www.scielo.org.co/scielo.php?pid=S0121$2562016000200002 \&$ script $=$ sci_abstract\&tlng $=e s$

7. Hernández-Lozano LR, Pulido-Caro AP. Estudio de parasitosis intestinal en niños pre-escolares del colegio anexo San Francisco de Asís-Bogotá. Pontificia Universidad Javeriana. Tesis de grado.2009;pag50. Disponible en:http:// hdl.handle.net/10554/8528 Minsalud. Encuesta Nacional de parasitismo intestinal en población escolar Colombia, 2012-2014. Universidad de Antioquia Instituto Nacional de Salud.2015. Disponible en: https://www.minsalud. gov.co/sites/rid/Lists/BibliotecaDigital/RIDE/VS/PP/ ET/encuesta-nacional-de-parasitismo-2012-2014.pdf

8. Pajuelo G, Luján D, Paredez B, Tello R. Aplicación de la técnica de sedimentación espontánea en tubo en el diagnóstico de parásitos intestinales. Rev Biomed [Internet]. 2006;17(2):96-101. Disponible en: http://www.medigraphic.com/pdfs/revbio/bio-2006/bio062b.pdf

9. Cardona J, Bedoya K. Frecuencia de parásitos intestinales y evaluación de métodos para su diagnóstico en una comunidad marginal de Medellín, Colombia. Latreia.2013;26:257-68. Doi: http://www.scielo.org.co/ pdf/iat/v26n3/v26n3a02.pdf

10. Garzón I, Flórez A, Ruda O, Reyes O. Evaluación del equipo FE-5 vs . técnica de Ritchie con muestras fecales de manipuladores de alimentos en cinco ciudades del país. Ciencias Biomed.2009;7(12):148-52. Disponible en: http://www.unicolmayor.edu.co/invest_nova/NOVA/ ART_ORIG4_12.pdf

11. Esenciales M. Instantáneas. Un nuevo método, más sensible, para detectar huevos de helmintos en heces. Rev Panamericana de Salud Pública.2002;12(2):123-7. Doi: http:// 
dx.doi.org/10.1590/S1020-49892002000800011

12. Aquino JM, Vargas GB, López B, Neri E, Bernal R. Comparación de dos nuevas técnicas de sedimentación y métodos convencionales para la recuperación de parásitos intestinales. medigraphic.org.mx.2012;59:233-42. Disponible en: http://www.medigraphic.com/pdfs/patol/ pt-2012/pt124i.pdf

13. Villafuerte-Ledesma KW, Noriega-Puga VR. Comparación de la técnica de Kato Katz vs la técnica del Mini Flotac para identificación de helmintos dentro del programa nacional para el abordaje multidisciplinario de parasitosis desatendias en el Ecuador. Universidad Técnica de Ambato. Tesis de Grado.2016;pag76. Disponible en: http://redi. uta.edu.ec/bitstream/123456789/23061/2/TESIS\%20 KELVIN\%20WLADIMIR\%20VILLAFUERTE\%20LEDESMA\%20PRIMERAS\%20HOJAS\%20CAMBIO.pdf

14. Chaguani-Cundinamarca. Sitio web del municipio Chaguaní en Cundinamarca [Internet]. Alcaldia de Chaguani.2015. Disponible en: http://www.chaguani-cundinamarca.gov.co/informacion_general.shtml\#geografia

15. Saldaña-Angel LH. Plan de desarrollo municipal 20122015 [Internet]. Chaguaní; 2015. Disponible en: Disponible en: http://cdim.esap.edu.co/BancoMedios/ Imagenes/chachaguanicundinamarcapd2012-2015.pdf

16. Fernández JA, Ramírez JD, López MC, Moncada LI, Reyes P, Heredia RD. Agreement of the Kato-Katz test established by the WHO with samples fixed with sodium acetate analyzed at 6 months to diagnose intestinal geohelminthes. ELSEVIER.2015;146:42-4. Doi: https://doi. org/10.1016/j.actatropica.2015.03.004

17. López MC, Moncada LI, Ariza-Araújo Y. Evaluación de tres pruebas para el diagnóstico de geohelmintos en Colombia. Rev Biomedica.2013;33:128-36. Doi: http://dx. doi.org/10.7705/biomedica.v33i1.633

18. Restrepo IC, Mazo LP, Salazar ML, Montoya MN, Botero JH. Evaluación de tres técnicas coproparasitoscópicas para el diagnóstico de geohelmintos intestinales. Latreia. 2012;26(1):15-24. Disponible en: http://www.scielo. org.co/pdf/iat/v26n1/v26n1a02.pdf

19. Gabrie JA, Rueda MM, Canales M, Sánchez A. Utilidad del método Kato-Katz para diagnóstico de UnciNarlaSis : experiencia en una zona rural de Honduras, 2011. Rev Medica Honduras.2012;80(3):96-101.Ddisponible en: http:// www.bvs.hn/RMH/pdf/2012/pdf/Vol80-3-2012-4.pdf

20. Navone G, Gamboa M, Kozubsky L, Costas M, Cardozo M, Sisliauskas M, et al. Estudio comparativo de recuperación de formas parasitarias por tres diferentes métodos de enriquecimiento coproparasitológico. Fed Latinoam Parasitológia.2005;60(1900):178-81. Doi: http:// dx.doi.org/10.4067/S0717-77122005000200014. Disponible en: http://www.scielo.cl/pdf/parasitol/ v60n3-4/art14.pdf

21. Rodríguez-Garcia AJ, Belmares-Taboada J, Hernández-Sierra JF. Factores de riesgo para oclusión y suboclusión intestinal por Ascaris lumbricoides.Rev Cir y Cir.2004;72(1):37-40. Disponible en: http://www.medigraphic.com/pdfs/circir/cc-2004/cc041h.pdf

22. Pinto-Rios CR, Peredo-Lazarte A. Obstruccion intestinal por ascaris lumbricoides. Rev Medica Cient Luz Vida.2010;1(1):36-40. Disponible en: http://www.imbiomed.com/1/1/articulos.php?method=showDetail\&id revista $=256 \&$ id_seccion $=4178 \& i d \_$ejemplar $=6937 \&$ id articulo $=69355$

23. Chacon-Fonseca N de J, Contreras R, Márquez W, Salinas RS, Romero JM. Importancia de la referencia médica en el diagnóstico de parasitosis intestinales por métodos coproparasitológicos. Rev de la Faculdad Med.2007;30(1):90-6. Disponible en: http:// www.scielo.org.ve/scielo.php?script=sci_arttext\&pid=S0798-04692007000100014

24. Carrada-Bravo T. Uncinariasis: ciclo vital, cuadros clínicos, patofisiología y modelos animales.medigraphic.org. mx.2007;54:187-99. Disponible en: http://www.medigraphic.com/pdfs/patol/pt-2007/pt074f.pdf

25. Peredo-Lazarte A, Carpio-Deheza G, Torrico-Rojas MC. Relación entre la presencia de anemia y la infección por uncinarias, en estudiantes del colegio "Ayopaya", Puerto Villarroel-Cochabamba. Rev Medica Cient Luz Vida.2012;3(1):21-6. Disponible en: http://www. imbiomed.com/1/1/articulos.php?method=showDetail\&id_articulo $=89227 \&$ id_seccion $=4176 \&$ id_ejemplar $=8772 \&$ id_revista $=256$

26. Espinosa M, Alazales M, García M. Parasitosis intestinal , su relación con factores ambientales en niños del sector "Altos de Milagro ", Maracaibo. Rev Cuba Med GenIntegr.2011;27(3):396-405. Disponible en: http://scielo.sld. $\mathrm{cu} / \mathrm{pdf} / \mathrm{mgi} / \mathrm{v} 27 \mathrm{n} 3 / \mathrm{mgi} 10311 . p d f$

27. Igual-Adell R, Domínguez-Márquez V. Estrongiloidiasis : epidemiología, manifestaciones clínicas y diagnóstico. Experiencia en una zona endémica : la comarca de La Safor (Valencia).Enferm Infecc Microbiol Clin.2007;25(3):3844. Doi:https://doi.org/10.1157/13111836

28. Carrada-bravo T. Strongyloides stercoralis: Cliclo vital, cuadros clínicos, epidemiología, patología y terapéutica. medigraphic.org.mx.2008;55:88-110. Disponible en: http://www.medigraphic.com/pdfs/patol/pt-2008/ pt082f.pdf

29. Giraldo JC, Pinzón LM, Rodríguez L, Vega V. Prevalencia de Enterobius vermicularis en niños en edad pre-escolar y escolar de la zona urbana del municipio de Chaguaní, Cundinamarca-Colombia en el primer trimestre del año 2015. Rev Visionarios en Ciencia y Téc- 
nología.2015;3(2):86-90. Disponible en: http://uroosevelt.edu.pe/assets/revista5_visionarios2016.pdf

30. López MC, Quiroz DA, Pinilla AE. Diagnóstico de amebiasis intestinal y extraintestinal. Acta Med Colombiana.2008;33(2):75-83. Disponible en: http://www.actamedicacolombiana.com/anexo/articulos/v33n2a6.pdf

31. Ximénez C, Morán P, Ramos F, Ramiro M. Amibiasis intestinal: estado actual del conocimiento. medigraphic.org.mx.2007;23(5):398-407. Disponible en: http:// www.cmim.org/boletin/pdf2007/MedIntContenido05_08.pdf

32. Ospina LC, Gómez LP, Vergara SS, Morales C, Consuegra $\mathrm{CP}$, Ríos KA, et al. Diagnóstico de parasitosis en estudiantes de la Institución Educativa de Ternera, Cartagena, 2010. Rev Fac de Ciencias de la Salud.2012;15-22. Disponible en: http://revistas.usb.edu.co/index.php/ Cienciactual/article/view/1592/2045

33. Giraldo JC, Riaño MM, Vásquez LR. Determinación de la seroprevalencia de cisticercosis porcina e identificación de teniasis humana en personas criadoras de cerdos en el área urbana del municipio de Coyaima Tolíma. Rev Med UMNG.2017;25(1):31-45. Doi: https://doi. org/10.18359/rmed.2916. Disponible en: https:// revistas.unimilitar.edu.co/index.php/rmed/article/ view/2916

34. Calchi M, Acurero E, Villalobos R, Colina M, Di Toro $\mathrm{L}$, Villalobos C. Comparación de técnicas de laboratorio para el diagnóstico de Giardia intestinales. Kasmera. 2014;42(1):32-40. Disponible en: http://www.scielo. org.ve/pdf/km/v42n1/art04.pdf

35. González AC, Giraldo JC. Prevalencia de parásitos intestinales zoonóticos en caninos (canis lupus familiaris) del área urbana del municipio de Coyaima (Tolima). Rev Med UMNG.2015;23(2):24-34. Doi: http://dx.doi. org/10.18359/rmed.1743. Disponible en: http:// revistas.unimilitar.edu.co/index.php/rmed/article/ view/1743/1403

36. Alvarado BE, Vásquez LR. Determinantes sociales, prácticas de alimentación y consecuencias nutricionales del parasitismo intestinal en niños de 7 a 18 meses de edad en Guapi, Cauca. Rev Biomédica.2006;26(1):81-91. Doi: https://doi.org/10.7705/biomedica.v26i1.1397 Disponible en: http://www.revistabiomedica.org/index. $\mathrm{php} /$ biomedica/article/view/1397

37. Ricaurte LA, Giraldo JC, Gutierrez E, Guatibonza AM. Estudio de parasitismo intestinal en caninos domiciliados en la zona urbana del municipio de Chaguaní Cundinamarca y posibles variables asociadas. Rev Visionarios en Ciencia y Técnología.2015;3(2):52-57. Disponible en: http://uroosevelt.edu.pe/assets/revista5_visionarios2016.pdf
38. Fernández J, Bernal MC, Giraldo JC. Parásitos gastrointestinales en caninos domiciliados en la Localidad de Teusaquillo (Bogotá-Colombia). Rev Cientifica Unincca.2015;20(1):67-72. Disponible en: https://www. unincca.edu.co/images/stories/pfd/revista-cientifica-v20-1.pdf

39. Díaz AM, Pulido MO, Giraldo JC. Nematodos con potencial zoonótico en parques públicos de la ciudad de Tunja, Colombia. Rev Salud Pública de Mexico.2015;57(2):170-176. Doi: http://dx.doi. org/10.21149/spm.v57i2.7413 Disponible en: http:// saludpublica.mx/index.php/spm/article/view/7413

40. Castañeda MA, Beltran KL, Moreno L, Peña LL, Riaurte JD, Giraldo JC. Estudio de parasitismo intestnal causado por protozoos en niños de dos a cinco años en un jardín infantil público de la Localidad de Chapinero en Bogotá, D.C. Rev Cientifica Unincca.2015;20(1):169-179. Disponible en: https://www.unincca.edu.co/images/stories/pfd/revista-cientifica-v20-1.pdf

41. Devera R, Cermeño J, Blanco Y, Bello M, Guerra X, De Sousa M, et al. Prevalencia de blastocistosis y otras parasitosis intestinales en una comunidad rural del Estado Anzoátegui, Venezuela. Fed Latinoam Parasitológia.2003;58:95-100. Doi: http://dx.doi.org/10.4067/ S0717-77122003000300001

42. Tarín L, Álvarez L, Chicue J, López D, Mendoza C. Parasitosis intestinal y factores de riesgo en niños de los asentamientos subnormales, Florencia- Caquetá, Colombia. Rev Nac Salud Pública.2015;33(2):171-80. Doi: http:// dx.doi.org/10.17533/udea.rfnsp.v33n2a04

43. Sorto ÓR, Portillo AM, Aragón MÁ, Saboyá MI, Ade MP, Minero MÁ, et al. Prevalencia e intensidad de la infección por geohelmintos y prevalencia de la malaria en escolares de El Salvador. Rev Biomedica.2015;35:407-18. Doi:https://doi.org/10.7705/biomedica.v35i3.2408

44. Londoño JC, Hernández AP, Vergara C, Matos R. Parasitismo intestinal en hogares comunitarios. municipio de Santo Tomas. Colombia, Atlántico. Rev Dugandia, Ciencias Básicas, Uniatlántico.2005;1(1):59-66. Disponible en: https://www.uniatlantico.edu.co/uatlantico/sites/ default/files/publicaciones/pdf/arc690_0.pdf

45. Giraldo MI, Lora F, Henao LH. Prevalencia de Giardiasis y Parásitos Intestinales en Preescolares de Hogares atendidos en un programa estatal en Armenia, Colombia. Rev Salud Pública.2005;7(3):327-38. Doi: http:// dx.doi.org/10.1590/S0124-00642005000300008

46. Mayorga Mogollón LE. Prevalencia de Parasitosis Intestinal en Consultantes al Hospital de Suaita-Santander. Salud UIS.2003;35:131-4. Disponible en: http:// revistas.uis.edu.co/index.php/revistasaluduis/article/ view/715/1001. 
47. Rodríguez-Saenz AY. Factores de riesgo para parasitismo intestinal en niños escolarizados de una institución educativa del municipio de Soracá - Boyacá. Rev Univ y Salud.2015;17(1):112-20. Disponible en: http://www. scielo.org.co/pdf/reus/v17n1/v17n1a10.pdf.

48. Alvarado M L, Hurtado A del P, Bernal M C. Preva- lencia de Giardia duodenalis y otros parásitos intestinales en niños del área urbana del municipio de Coyaima, Tolima (Bogotá-Colombia). Rev Cientifica Unincca.2015;20(1):75-85. Disponible en: https:// www.unincca.edu.co/images/stories/pfd/revista-cientifica-v20-1.pdf 